\title{
Learning in English Using Mobile Social Media Applications: A Case Study in Saudi Higher Education
}

\author{
Abdulrahman Alshabeb \\ University of Wolverhampton
}

\begin{abstract}
Mobile smartphones and social media applications have become a vital personal and business tool of the 21 st Century in the maintaining of contact, intercommunication, organisation of appointments, entertainment, business and education, deemed by users indispensable to the demands of modern living. In essence, age is no determinant in the use of the digital technology. Saudi Arabia has a substantial young population who are becoming more digitally competent and increasingly connected with each other, especially through social media tools such as WhatsApp, Twitter, YouTube, and Snapchat (GMI, 2018). The purpose of this study is to harness their ubiquitous use of social media in the pursuit of their language education. The purpose of this study is to explore how mobile devices and social media applications can promote and provide more collaborative and contextual learning experiences. This study is grounded in the tenets of the connectivism learning theory. a design-based research approach was implemented to allow for more rigorous design principles for mobile language learning. Qualitative research based on focus groups interviews with Saud Arabian language students of both genders were the basis sample method of this study. The study has drawn upon the functionality of design-based research to identify appropriate design principles that can be employed for mobile language learning.
\end{abstract}




\section{IA C EDUCATION}

International Academic Conference on EDUCATION

This study has investigated the potential of mobile phones and mobile social networking in maintaining effective learning environment, and explored whether they can assist EFL students in establishing a collaborative mobile medium that takes advantage of students' familiarity with the use of mobile phones, on the one hand, and social networking environments such as WhatsApp, on the other. The findings from the current study has significantly extended the understanding of mobile learning, showing it can provide

rich out-of-class learning opportunities in contexts that are characterised by limited language learning opportunities and socio-cultural restrictions to face-to-face student interactions. Students also co -contributed to the design adjustment and were happy to experience that adjustment in their actual learning. Finally, this study can provide further evidence of the possibility as well as the effectiveness of 'reforming' EFL education in Saudi Arabia using students' voices to enhance their contribution to the learning process.

Keywords: Collaborative Leaning; Connectivism; MALL; Social Media; Design Principles 